\title{
Experiencia académica en desarrollo rápido de sistemas de información web con Python y Django
}

\author{
Cristian L. Vidal-Silva ${ }^{1 *}$, Aurora Sánchez-Ortiz' ${ }^{1}$, Jorge Serrano ${ }^{1}$ y José M. Rubio ${ }^{2}$ \\ (1) Departamento de Administración, Facultad de Economía y Administración, Universidad Católica del Norte, Angamos \\ 0610, Antofagasta - Chile (correo-e: cristian.vidal@ucn.cl; asanchez@ucn.cl; jorge.serrano@ucn.cl). \\ (2) Escuela de Computación e Informática, Facultad de Ingeniería, Ciencia y Tecnología, Universidad Bernardo \\ O’Higgins, Avenida Viel 1497, Ruta 5 Sur, Santiago - Chile, (correo-e: josemiguel.rubio@ubo.cl).
}

* Autor a quien debe ser dirigida la correspondencia

Recibido Mar. 4, 2021; Aceptado May. 4, 2021; Versión final Jun. 16, 2021, Publicado Oct. 2021

\begin{abstract}
Resumen
Este trabajo presenta la experiencia académica, tanto de profesores como estudiantes, en un curso de último año para el desarrollo de sistemas de información web mediante el uso del marco de trabajo Django del lenguaje de programación Python. Django facilita el desarrollo de aplicaciones web con el uso del patrón de desarrollo Modelo-Plantilla-Vista (MTV, en inglés). Por la simplicidad sintáctica y práctica de Python y Django, los estudiantes de una carrera del área de administración y ciencias de la información fueron capaces de desarrollar prototipos de sistemas de información. Las experiencias de los estudiantes permiten remarcar la relevancia de las primeras fases del ciclo de desarrollo de software, requerimientos y diseño. Los proyectos que se presentan son sistemas web prototipo con operaciones para crear, leer, actualizar y eliminar (CRUD, en inglés) registros de la base de datos. Se concluye que Python es un lenguaje de programación adecuado para un fácil y rápido dominio.
\end{abstract}

Palabras clave: Python; Django; MTV; software; desarrollo

\section{Academic experience in rapid development of web information systems with Python and Django}

\begin{abstract}
This work presents the academic experience, both for teachers and students enrolled in a last year course, in developing web information systems by using the Django framework of the Python programming language. Django facilitates developing web applications by using the Model-Template-View (MTV) development pattern. Due to the syntactic and practical simplicity of Python and Django, students enrolled in the management and information science degree are able to develop prototypes of information systems. The students' experiences allow highlighting the relevance of the initial phases of software development cycle, requirements, and design. The projects presented here are prototype web systems with operations to create, read, update, and delete (CRUD) records from a database. It is concluded that Python is an adequate programing language because of its ease and rapid mastering.
\end{abstract}

Keywords: Python; Django; MTV; software; development 


\section{INTRODUCCIÓN}

El uso de Tecnologías de la Información (TI) es parte de la cotidianidad de las personas. Las $\mathrm{TI}$ juegan un papel fundamental para las organizaciones hoy en día, para que estas puedan competir en el mercado global (Oliva et al., 2020). Los sistemas de información web representan un activo de alto orden para las organizaciones. Tal y como señala (Aguadero et al., 2015), una aplicación web es básicamente una manera de facilitar el logro de una tarea específica en la Web, mientras un sitio web estático esencialmente es una herramienta para la comunicación. Los sistemas de información web son ejemplos de aplicaciones web que han evolucionado desde páginas web dinámicas hasta sistemas de información con bases de datos (Zheng et al, 2021). En Chile y el mundo, hay una tendencia en la educación superior hacia la definición de carreras profesionales para el desarrollo de competencias que antes eran poco comunes (Schmal et al., 2020). Un ejemplo de estas carreras resulta ser Ingeniería en Información y Control de Gestión (IICG) (IICG-UCN, 2021) cuyo foco es desarrollar competencias de ciencias de la información, administración de empresas y control de gestión.

El objetivo de este trabajo es presentar experiencias tanto de los estudiantes como de académicos de IICG con el uso del marco de trabajo Django en Python que permite el desarrollo rápido de sistemas de información web. Python es un lenguaje de programación de alto nivel, interpretado, de una sintaxis sencilla, propiedades que lo hacen muy adecuado para el proceso de enseñanza-aprendizaje de lenguaje de programación (Vidal et al., 2010; Koulouri et al., 2015; Shukla y Parmar, 2016). La figura 1 muestra el clásico ejemplo del mostrar un mensaje en Python, y se aprecia que sólo es necesaria la orden o instrucción para escribir o mostrar un mensaje en la pantalla (print) además del mensaje que se desea mostrar. Este ejemplo fue implementado con la herramienta en línea Google Colaboratory (Carneiro et al., 2018). Python también soporta características de computación moderna tales como grandes datos (Big Data en inglés), aprendizaje de máquina (Chang et al., 2018), y el desarrollo de aplicaciones web con poca programación como con el marco de trabajo Django (Melé, 2020). Django muestra la relevancia de los requerimientos en el ciclo de desarrollo de software como fuente de un buen diseño e implementación de soluciones software.

Actualmente, el mundo vive en una sociedad de información. En este contexto, las instituciones de educación superior presentan carreras profesionales para desarrollar competencias de ciencias de la información junto con alguna otra área del saber tal como administración y control de gestión (IICG-UCN, 2021). Este trabajo describe una experiencia pedagógica en un curso de último año de IICG (2021), esto es, en el curso Desarrollo de SIA de IICG en la sede Antofagasta de la Universidad Católica del Norte (UCN) durante el primer semestre del año 2020.

La carrera de IICG en la UCN fue creada en el año 2001 para la formación de profesionales con una sólida formación en ciencias de la administración y de la información. Dentro de su plan de formación actual, los estudiantes reciben una mayor formación en el área de negocios. Según la malla curricular actual de IICG (Malla-IICG, 2021), el primer curso de ciencias de la información es Herramientas de Programación en el quinto semestre del plan curricular. El curso Desarrollo de SIA es un curso del noveno semestre de IICG, cuyos principales objetivos de aprendizaje son comprender y aplicar las fases de un ciclo de desarrollo de software tradicional, esto es, especificación y requerimientos de software, diseño de software, implementación y prueba de software, mantención de software.

En este contexto, dada la formación previa en cursos del área de ciencias de la información, es necesario aplicar herramientas que soporten el desarrollo rápido de aplicaciones software para minimizar los costos de programación. La tabla 1 describe los principales cursos del área de ciencias de la información y tecnología de la malla curricular actual de IICG junto con el semestre académico de los mismos. Como se aprecia, el primer curso de ciencias de la información y tecnología es del 5to semestre de formación. Justamente, durante 2022 IICG UCN presentará una nueva malla curricular con la inclusión de cursos del área de ciencias de la información y tecnología desde el primer semestre y durante todo su plan de formación.

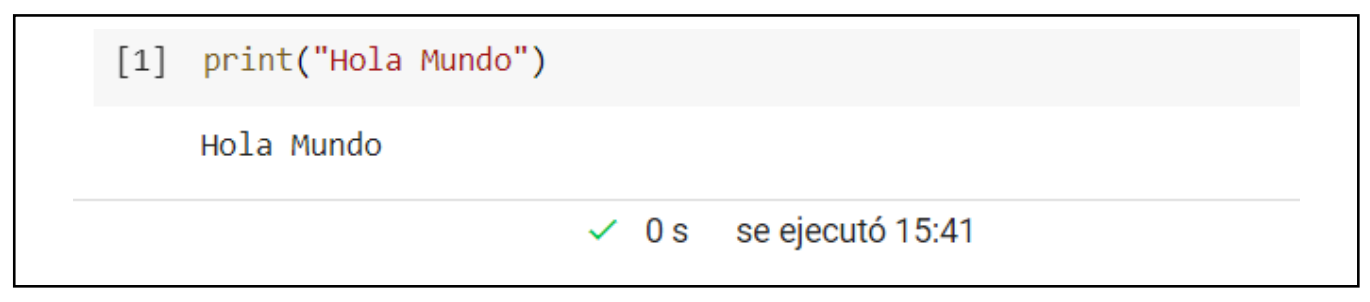

Fig. 1. Hola Mundo en Python. 
Tabla 1: Cursos de Ciencias de la Información y Tecnología de Malla Curricular IICG-UCN.

\begin{tabular}{|l|l|c|}
\hline \multicolumn{1}{|c|}{ Curso } & \multicolumn{1}{|c|}{ Objetivo General } & $\begin{array}{c}\text { Semestre } \\
\text { Académico }\end{array}$ \\
\hline Herramientas de Programación & $\begin{array}{l}\text { Este curso está dirigido a estudiantes sin experiencia en } \\
\text { programación. Su objetivo general es entregar los } \\
\text { fundamentos para entender la programación de } \\
\text { computadores. En este curso se utiliza el lenguaje de } \\
\text { programación Python. }\end{array}$ & $\mathrm{V}$ \\
\hline Gestión de Base de Datos & $\begin{array}{l}\text { Aplicar técnicas de modelamiento de datos para la gestión } \\
\text { de la información en la organización. }\end{array}$ & VI \\
\hline Elementos Comunicacionales & $\begin{array}{l}\text { Identificar y aplicar los conceptos y principios } \\
\text { fundamentales de la construcción de redes } \\
\text { comunicacionales. }\end{array}$ & $\begin{array}{l}\text { VII } \\
\text { gestión e implementación de negocios electrónicos. }\end{array}$ \\
\hline e-Business & $\begin{array}{l}\text { El curso permitirá conocer y utilizar las metodologías y } \\
\text { herramientas de análisis, diseño e implementación de } \\
\text { sistemas de información para apoyar la gestión } \\
\text { organizacional. }\end{array}$ & VIII \\
\hline Desarrollo de SIA & $\begin{array}{l}\text { El curso permitirá al estudiante conocer elementos de } \\
\text { ciencias de datos y su aplicación en un contexto } \\
\text { empresarial. }\end{array}$ & $\mathrm{IX}$ \\
\hline Taller Aplicado
\end{tabular}

\section{OTROS ANTECEDENTES}

A continuación, se describen el desarrollo rápido de aplicaciones software (RAD del inglés Rapid Application Development), Python y el marco de trabajo Django.

\section{Desarrollo rápido de aplicaciones $R A D$}

RAD representa un enfoque de desarrollo de software adaptativo con menos énfasis en la planificación del desarrollo y más énfasis en la producción y adaptación de prototipos (Poulovassilis et al., 2019). Las fases de RAD son: i) Planificación de requerimientos, ii) Diseño del usuario, iii) Construcción, iv) Transición. Tal y como se aprecia en la figura 2, RAD es análogo a un enfoque de desarrollo evolutivo. Tal como señala Zheng et al. (2021), existe una evolución de los sistemas de Software con la expansión de Internet en el mundo. Ajustes de arquitectura de los sistemas de información web para satisfacer requisitos variantes y focos de mayor amplitud. En dicho contexto, son necesarios lenguajes de programación y/o marcos de trabajo que permitan un desarrollo rápido de sistemas de información.

\section{Python y Django}

Python es un lenguaje de programación libre, de alto nivel y multiplataforma inventado por Guido Van Rossum en 1989 (Sánchez-Carrión et al., 2018); es decir, Python funciona sin costo es Windows, Unix, Linux, y otros sistemas operativos con una sintaxis más simple y elegante que la de otros lenguajes de programación (Prokopyev et al., 2020). Django es un marco de trabajo (framework) para el desarrollo de aplicaciones web usando Python. Django considera algunas funcionalidades listas para usar para facilitar el desarrollo de aplicaciones web. Como resultado, no es necesario escribir todo el código ni usar tiempo para buscar errores de código en el framework. Es decir, mediante Django, el desarrollo de sistemas de información web puede ser rápido, seguro, escalable y también fáciles de mantener (Salas-Zarate et al., 2015; Sánchez-Carrión et al., 2018). Django representa un marco de trabajo para el desarrollo rápido de sistemas de información web con Python.

Los marcos de trabajo de desarrollo web tales como PHP Codelgniter (Haristiani, N. y Rifa'i, 2020) y PHP Kapoor (2020) soportan un patrón de diseño MVC (Model-View-Controller) de arquitectura de software para desarrollar aplicaciones web. Django implementa el concepto de MTV (Model-Template-View) que es ligeramente diferente de MVC. Las figuras 3 y 4 (Rosenbloom, 2018) ilustran los principales componentes de los patrones de diseño MVC y MTV, respectivamente, y las principales relaciones entre dichos componentes. 


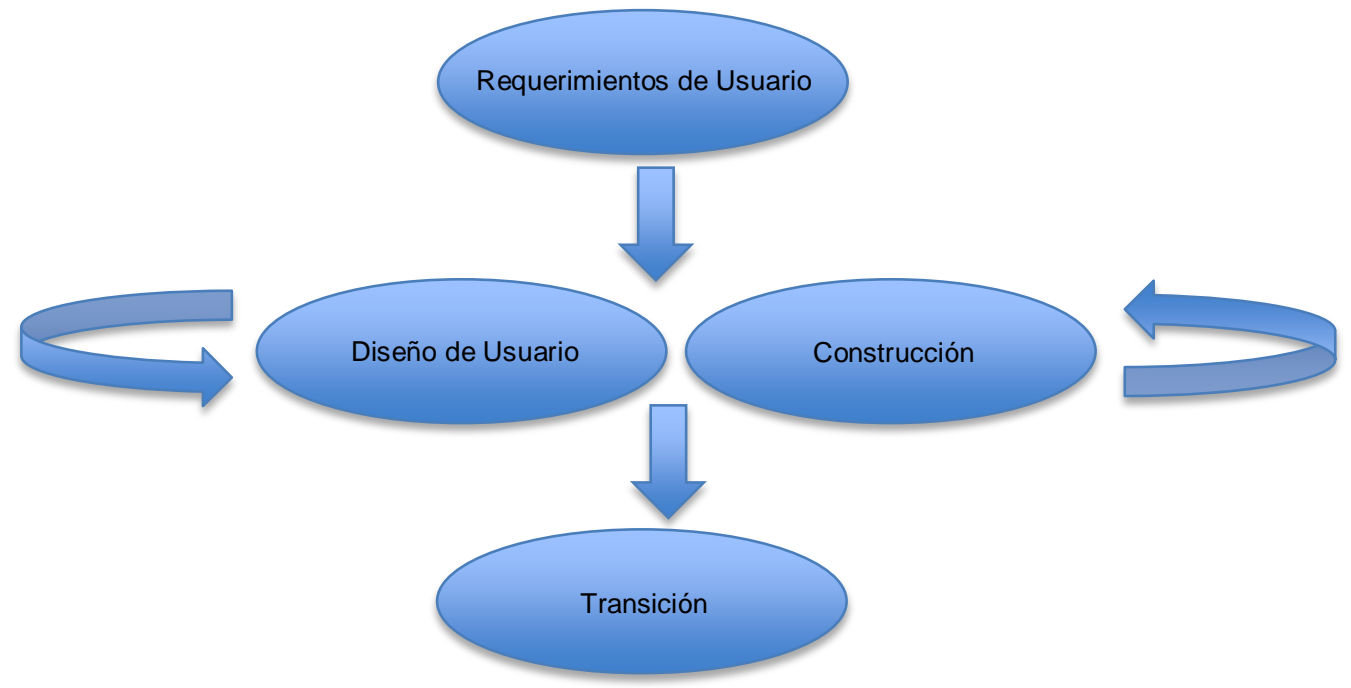

Fig. 2. Fases de RAD.

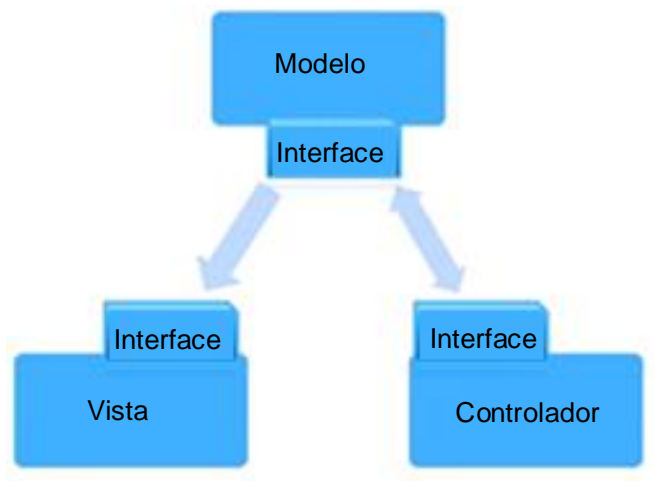

Fig. 3. Componentes de patrón de diseño MVC.

MVC define un aislamiento y separación de incumbencias entre la lógica de aplicación (modelo) y la interfaz de usuario (datos): el controlador recibe todas las solicitudes desde la aplicación y luego trabaja con el modelo para preparar los datos necesarios para la vista. Luego la vista usa los datos preparados por el controlador para generar una respuesta final (Rosenbloom, 2018). En ambos patrones, el modelo representa la capa de acceso a datos. La vista en MVC representa la interfaz de usuario, mientras la plantilla de MTV representa la interfaz de usuario. El controlador de MVC es el encargado de definir la lógica de negocio, mientras en Django la vista representa una capa intermedia para definir dicha lógica. La principal diferencia entre MVC y MTV es que, en este último, Django se encarga de tareas propias del controlador (para controlar las interacciones entre el modelo y la vista).

Como se ilustra en la figura 4, Django posibilita definir proyectos y aplicaciones para sistemas de información web. Cuando se define un proyecto Django, automáticamente se genera una serie de archivos, y uno de los más relevantes es settings.py. El archivo setting.py se utiliza para definir variables de configuración del sistema web tales como las aplicaciones que incluye, parámetros de conexión a base de datos, idioma, entre otros. Una vez que se define un proyecto Django, es posible definir aplicaciones para dicho proyecto. Para cada aplicación de proyecto Django, se pueden definir modelos que representan tablas en la base de datos establecida (archivo models.py) junto con parámetros de visualización de estos modelos. Tal y como resaltan Melé (2020) y Zheng et al. (2017), Django permite, de manera predeterminad, utilizar una capa de mapeo relacional de objetos (ORM del inglés Object Relational Model) para la interacción con aplicaciones Django con bases de datos relacionales. Además, en un proyecto Django se pueden incluir plantillas con extensión .html para la visualización de datos (archivos .html en carpera templates), además de plantilla de administración para operaciones CRUD (del inglés Create, Read, Update y Delete) para los modelos (tablas) que se indiquen en el archivo admin.py. Ser herramientas de desarrollo del mundo libre o código abierto (del inglés open source), además de su simplicidad para desarrollar soluciones, hacen muchos desarrolladores en el mundo usen y desarrollen extensiones para estas herramientas. Además, por su licencia libre, existen servidores de libre acceso como Django (Danielsson et al., 2021), que de manera simple permiten alojar y acceder sin restricciones a soluciones Django. 


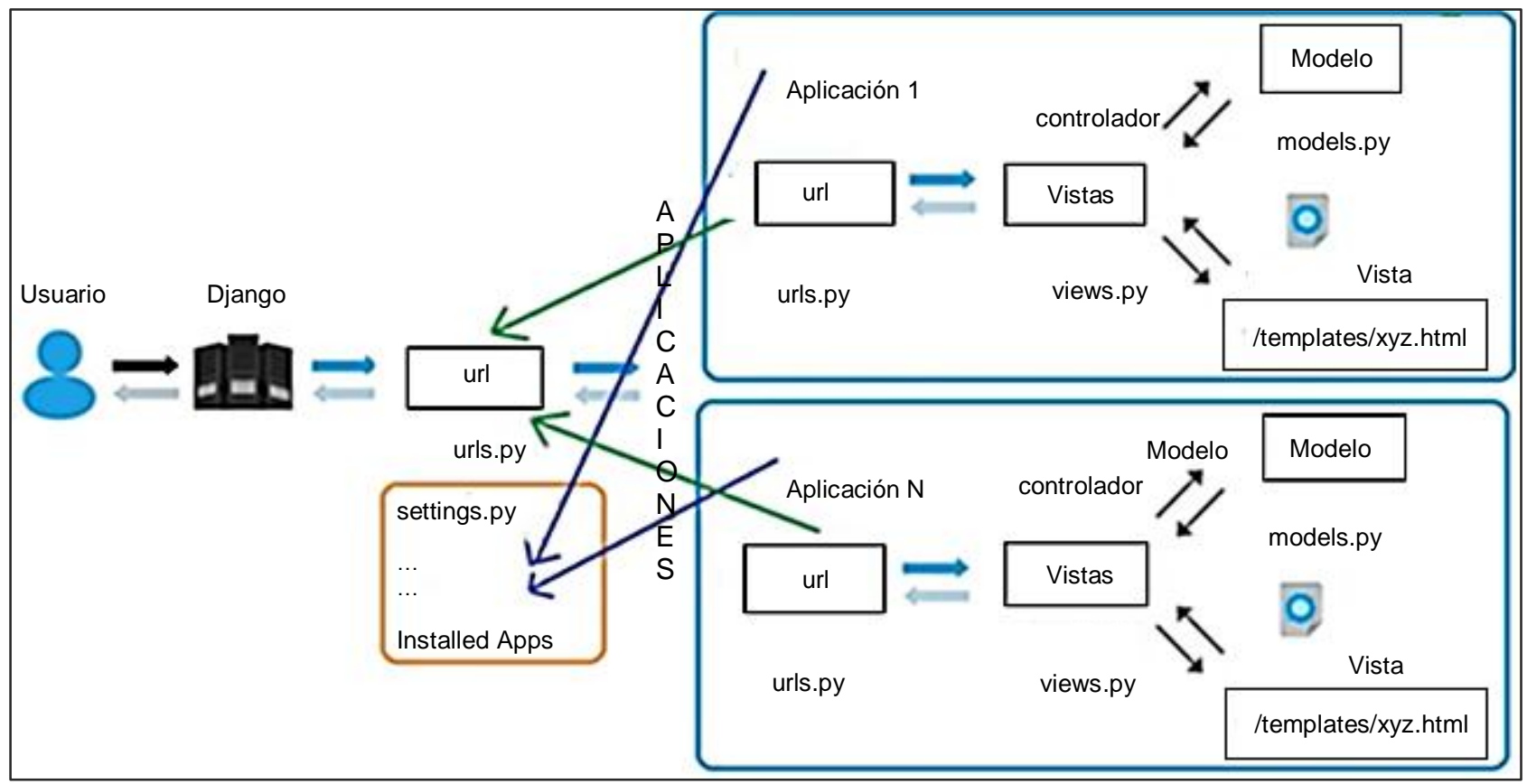

Fig. 4. Componentes de patrón de diseño MTV en aplicación Django.

\section{METODOLOGÍA}

Durante el primer semestre del año 2020, bajo una modalidad en línea por los devastadores efectos a nivel global de la pandemia de COVID 19 (Gaudiot y Kasahara, 2020), se procedió al desarrollo de proyectos en el curso Desarrollo de SIA del noveno semestre de IICG. A continuación, se detallan 2 proyectos ejemplo desarrollados por grupos de estudiantes durante el indicado periodo académico. Existe una completa hegemonía entre un diagrama de clases y el modelo Django. Django permite operaciones CRUD (Seo et al., 2017) con un costo de desarrollo mínimo; esto es, con la definición de clases del modelo, y luego la configuración de archivo de administración para la visualización de clases para un usuario administrador. Por el contexto del tiempo y la modalidad del año académico 2020, y para demostrar la simplicidad de desarrollar aplicaciones con operaciones CRUD Django, durante parte del primer semestre académico 2020, esto es, desde la última semana de Junio a primera semana de Agosto de 2020, los estudiantes tuvieron lecciones del tema para llevar a cabo el desarrollo de proyectos. A continuación, se describe dos de estos proyectos, además de dar a conocer una tabla del estado de cada uno de estos.

\section{Sistema de venta de artículos electrónicos}

El primer ejemplo de sistema CRUD Django es un sistema web para la venta de artículos electrónicos. Como se aprecia en la figura 5, este sistema considera una clase para Cliente con una subclase para Cliente Preferente. La clase Cliente tiene una asociación de 1 a muchos con la clase Artículo, esto es, una instancia de cliente puede reservar entre 1 y muchas instancias de artículo. Un cliente puede recibir múltiples órdenes de despacho según los artículos reservados por el cliente. Existe una herencia de clase Artículo (súper clase) y artículos computacionales (A. Computacionales), artículos electro-hogar (A. ElectroHogar), y artículos de televisión y audio (A. TvAudio). Cada instancia de la clase cliente puede tener 1 o múltiples instancias de Sistema Bancario. Este diagrama de clases respeta el principio de ocultamiento de información (Leavens y Muller, 2007). La figura 6 muestra parte del archivo de aplicación models.py para la definición de los modelos (tablas) del sistema web para la venta de artículos electrónicos. Nótese que un sistema Django no requiere el uso directo de SQL para la definición, creación y manipulación de datos.

\section{Sistema de venta de joyas}

El segundo ejemplo de sistema CRUD Django es un sistema web para la venta de joyas. Como muestra la figura 7, este sistema considera una clase Cliente, una clase Vendedor, y una clase Pedido que están relacionadas, esto es, una instancia de Cliente puede generar uno o muchos pedidos, mientras un vendedor recibe uno o muchos de estos pedidos. Se asume que cada instancia de pedidor contiene se relaciona a una o muchas instancias de joyas, donde cada joya está asociada a una categoría, y cada Categoría puede asociarse a una o muchas joyas. Nótese que este diagrama de clases es de muy alto nivel ya que no considera detalles de ocultamiento de información (Kaw et al., 2019), y detalles de multiplicidad para nuevas instancias (por ejemplo, cuando se crea un nuevo vendedor y/o cliente, no inmediatamente están relacionados a un pedido). 


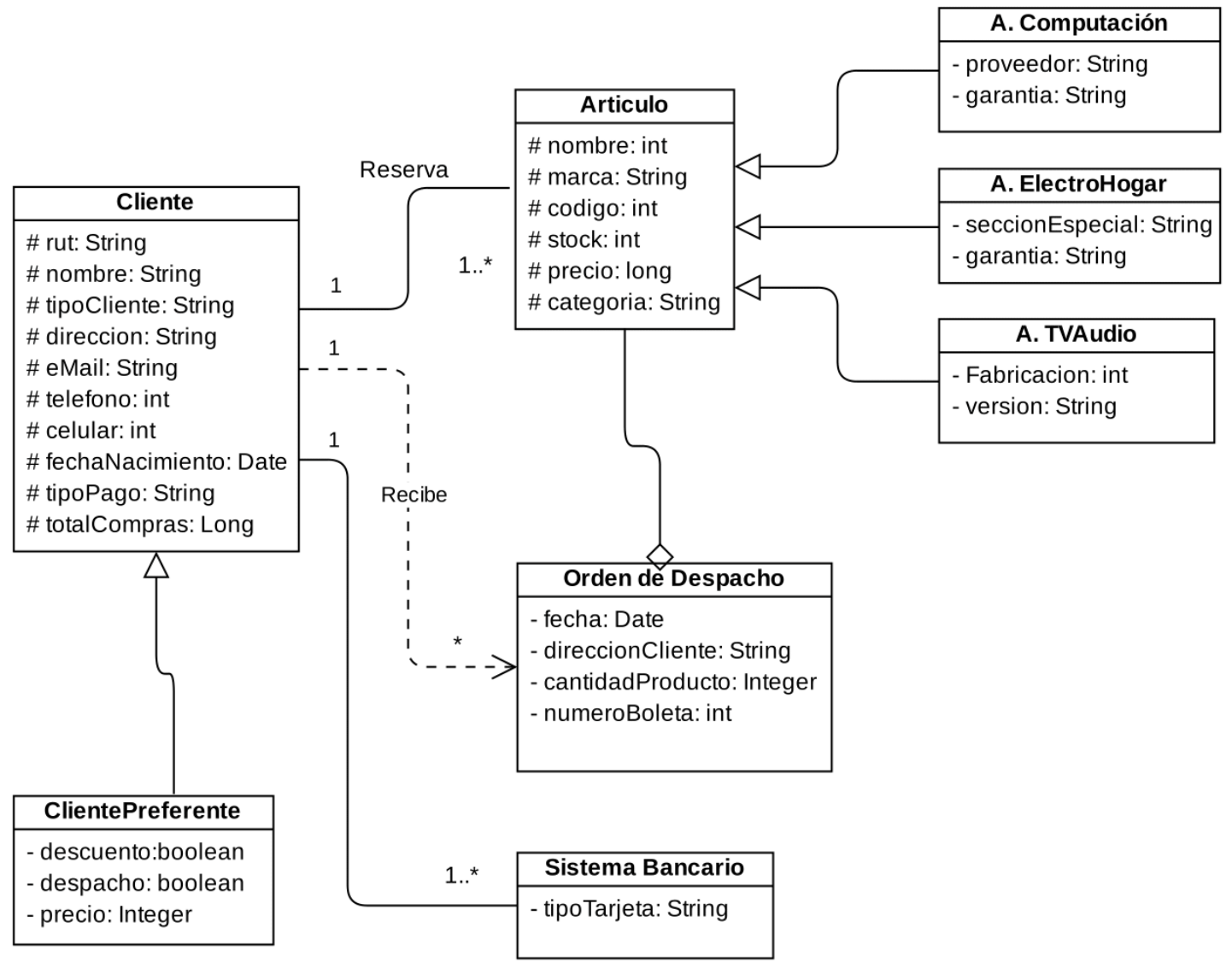

Fig. 5. Diagrama de clases de sistema web para la venta de artículos electrónicos IICG 2020.

Fig. 6. Clases (tablas) de archivo models.py de sistema web para la venta de artículos electrónicos IICG 2020.

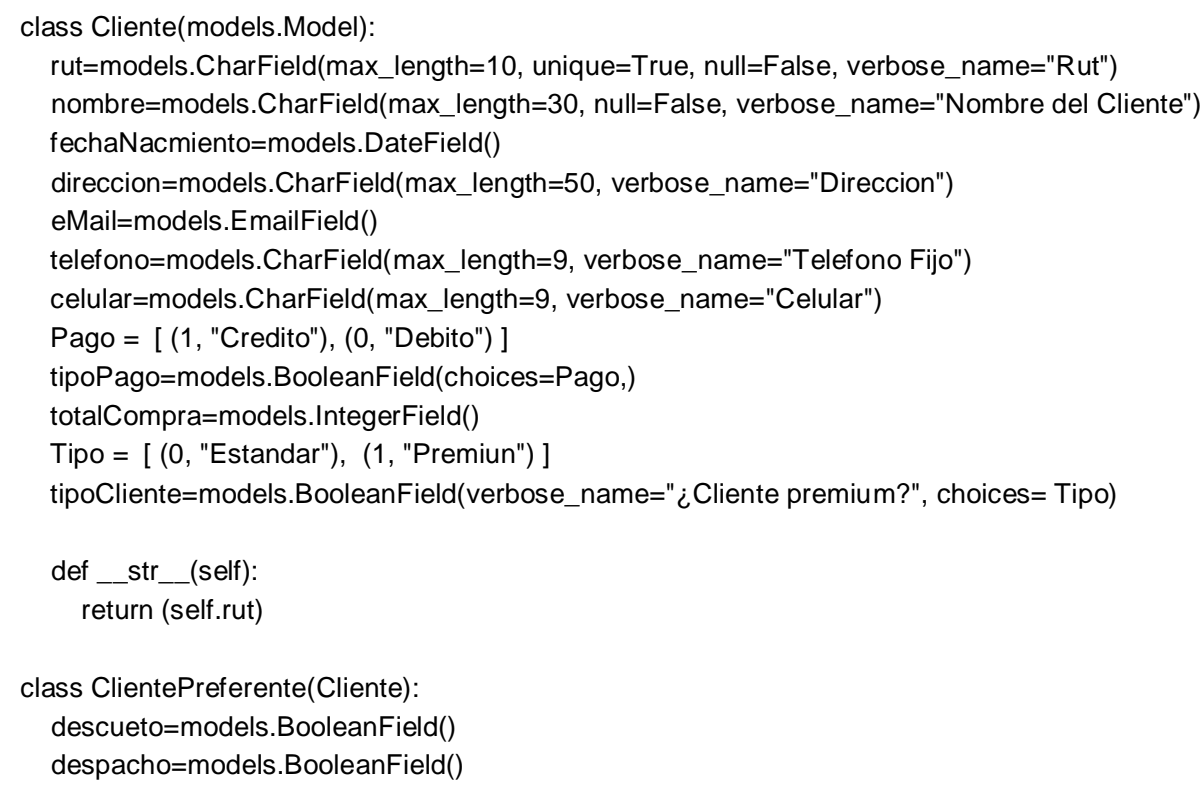




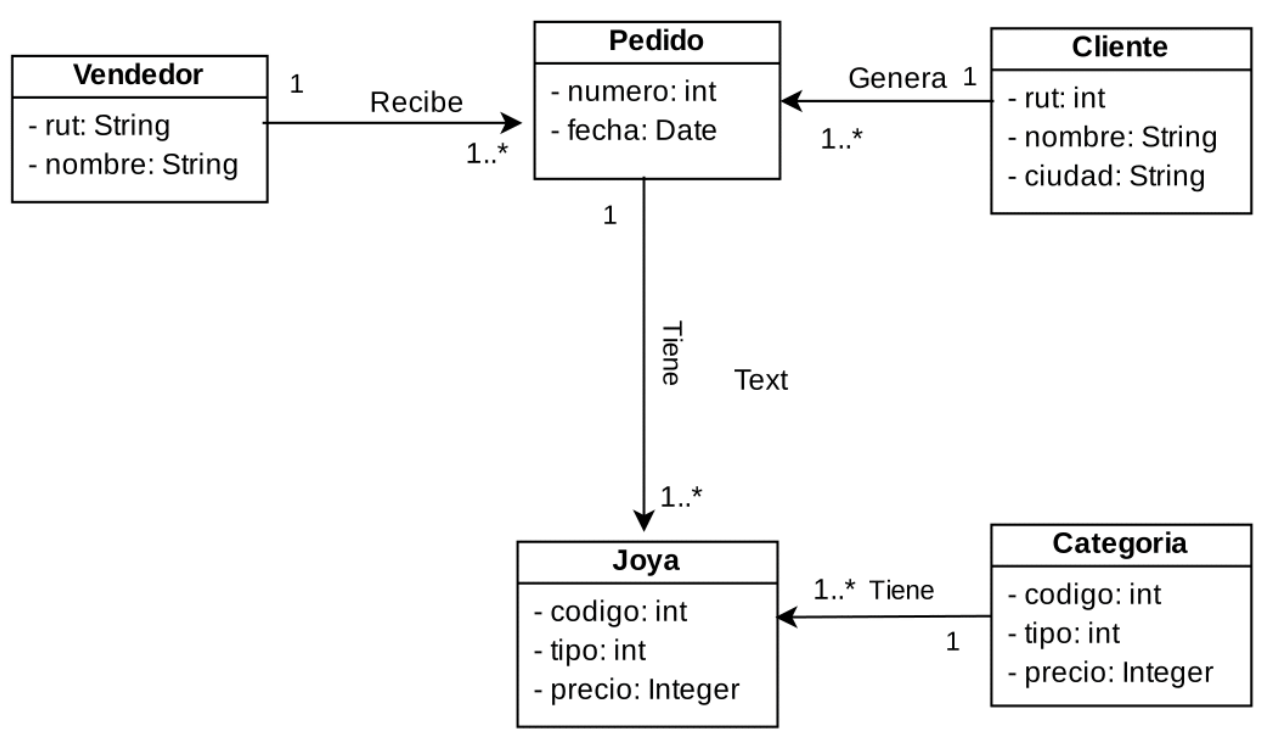

Fig. 7. Diagrama de clases de sistema de información web para la venta de joyas IICG 2020.

\section{RESULTADOS}

La tabla 2 presenta detalles de los 7 proyectos desarrollados en el curso Desarrollo de SIA de IICG, esto es, título del proyecto, número de integrantes, sistema de base de datos utilizado, y su estado final. Tal y como se aprecia, 4 proyectos usaron PostgreSQL, 3 proyectos usaron MySQL y uno de ellos SQLite 3 como sistema gestor de base de datos. Sólo 1 proyecto no llegó a estar 100\% funcional que se puede deber a la falta de trabajo en equipo. Había libertad para la conformación de los grupos con un máximo de 6 integrantes. Durante el desarrollo de estos proyectos, era necesaria la presentación de dos avances para dar a conocer tanto los requerimientos como el diseño de la solución, aunque finalmente sólo 1 de ellos fue efectivo, además de la entrega final. Durante 4 semanas, se realizaron en total 8 clases acerca de Python y Django. Cada clase es de 1 hora y 30 minutos; es decir, fueron 12 horas de clases en total. Estas clases fueron realizadas desde la última semana de junio hasta la primera semana de agosto de 2020 (hubo 2 sesiones para presentar el 1er avance y la entrega final de los proyectos). Todas las clases fueron en modalidad en línea mediante plataforma Zoom dada la situación de pandemia en Chile y el mundo, y, estas fechas, producto de una paralización estudiantil durante los primeros meses académicos de 2020 (mayo y junio).

\section{Administración de Django}

\section{Sitio administrativo}

\begin{tabular}{lcc}
\hline APLICACION1 & + Añadir & Modificar \\
\hline Categorias & + Añadir & Modificar \\
\hline Clientes & + Añadir & Modificar \\
\hline Joyas & + Añadir & Modificar \\
\hline Pedidos & + Añadir & Modificar \\
\hline Vendedores & & Mñ
\end{tabular}

AUTENTICACIÓN Y AUTORIZACIÓN
\begin{tabular}{ll} 
Grupos & + Añadir Modificar \\
\hline Usuarios & + Añadir Modificar
\end{tabular}

Fig. 8. Panel de administración Django de sistema de información web para la venta de joyas IICG 2020. 
La figura 8 muestran la interfaz Django para la administración de registros de Categoría, Cliente, Joyas, Pedido y Vendedor para realizar operaciones CRUD sin costos de implementación de las clases del modelo, un gran beneficio para la disminución de costos de desarrollo, y elevar la importancia que conlleva un buen análisis de requerimientos y modelamiento estructural del sistema de información a ser desarrollado. Django ofrece un sistema de administración para realizar operaciones CRUD en cada una de las clases (tablas) que se indique, además de un sistema propio de usuarios para la operación. Cada una de estas tablas se listan en orden ascendente alfabéticamente y se les agrega una letra s según su definición en el archivo de modelos (model.py), aunque todos estos valores son configurables en cada uno de los modelos.

Tabla 2: Proyectos Django Desarrollo de SIA IICG 2020.

\begin{tabular}{|l|c|l|c|}
\hline \multirow{2}{*}{\multicolumn{1}{|c|}{ Título Proyecto }} & \multicolumn{3}{c|}{ Proyectos Diango Desarrollo de SIA IICG } \\
\cline { 2 - 4 } & \# Integrantes & SGBD & Estado Final \\
\hline Sistema de joyería & 3 & Postgresql & OK \\
\hline Sistema de post-venta & 6 & Sqlite3 & OK \\
\hline Sistema de artículos electrónicos El Futurista & 4 & Postgresql & OK \\
\hline Sistema de pizzería La Gatita & 3 & Mysql & OK \\
\hline Sistema de ventas & 6 & Postgresql & OK \\
\hline Sistema de Hotel Pichichi & 4 & Mysql & OK \\
\hline Sistema de clases particulares & 5 & Postgresql & OK \\
\hline Sistema de clases online & 1 & MySQL & -- \\
\hline
\end{tabular}

Los resultados de la tabla 2 avalan el potencial de desarrollo rápido de sistemas de información web del marco de trabajo Django, más aún si se considera el actual soporte de servidores web libres como Heroku (Danielsson et al. 2020) para desarrollar y alojar sistemas web Django sin costo. En la versión 2021 del curso de Desarrollo de SIA de IICG, el cual se dictará en línea y al mismo tiempo para las sedes Antofagasta y Coquimbo de la UCN, se trabajará para la publicación de cada uno de los proyectos en Heroku con la metodología aprendizaje + servicio UCN (CIMET, 2021) para desarrollar soluciones a socios comunitarios. Es importante señalar que el año anterior, los proyectos fueron con OpenXava (Marties, 2014) en 16 semanas con un desarrollo exitoso del $70 \%$ de estos (7 de 10). Es decir, el desarrollo Django Python fue realmente superior en tiempo y efectividad en el desarrollo de proyectos.

\section{DISCUSIÓN}

En un contexto de competencias y aprendizaje para carreras profesionales de las áreas de ciencias de la información y ciencias de la administración, el trabajo de (Tapia-Jara et al., 2020) describe experiencias de autoaprendizaje de estudiante de IICG con el uso de plataformas Youtube. Por otra parte, en el trabajo de Schmal (2015), se describen experiencias universitarias para la definición de competencias genéricas con especialización para Ingeniería Informática Empresarial de la Universidad de Talca (IIE-UTAL). Schmal et al. (2020) resume los principales asuntos para la definición de programas de estudio cuya meta es desarrollar competencias genéricas, con ejemplos de IIE-UTAL. Respecto al uso de Python para el desarrollo de competencias y habilidades de programación, Gutiérrez-Cárdenas (2020) describe experiencias del uso de lenguaje de programación Python en curso de programación para estudiantes del área de negocios. Así mismo, el trabajo de Vidal et al. (2010) ejemplifica experiencias y resultados de aprendizaje con estudiantes de curso de primer año de Ingeniería Informática Empresarial durante el año 2010 en la Universidad de Talca. El trabajo de Tabet et al. (2016) describe experiencias de desarrollo de competencias de programación usando Python pero con jóvenes de enseñanza media en Qatar. Considerando experiencias pedagógicas con Django, Rainka et al. (2016) describe positivas experiencias de aprendizaje activo de estudiantes en un curso de Internet de las cosas donde una de las herramientas utilizadas es Django para el desarrollo rápido de aplicaciones web.

Enfrentados al desafío de desarrollar competencias de ciencias de la información en carreras de negocios, las carreras y profesores necesitan innovar en las herramientas y metodologías de enseñanza. Para abordar esta limitación, se ha logrado con éxito el objetivo general de este estudio al presentar experiencias de estudiantes y académicos en el uso de Django y Python. Django es un marco de trabajo que permite dar una gran importancia a las fases de requerimientos y diseño de software, para garantizar así el desarrollo de sistemas web de calidad. Gracias a la simplicidad y actualidad de la sintaxis de Python, el uso del marco de trabajo Django permitió el desarrollo de sistemas CRUD por estudiantes con pocas competencias de lenguajes de programación y Python. Este trabajo agrega conocimiento para trabajos futuros que exploren el comportamiento de los estudiantes frente a nuevas metodologías y herramientas para el desarrollo de competencias de ciencias de la información. Aporta a las instituciones educativas la utilización de un marco 
de trabajo para la enseñanza basado en el desarrollo rápido de proyectos de sistemas de información en carreras de negocios. Finalmente, esta experiencia pude ser replicada en distintas instituciones y carreras.

La experiencia lograda con los estudiantes de IICG UCN y Django es una invitación para la adopción de Python como lenguaje de programación oficial en módulos del área de ciencias de la información. Estos resultados se deben ver en el contexto de sus limitaciones. Primero, la experiencia cuenta con la participación de estudiantes de un curso de la carrera, por lo que no se ha podido realizar comparaciones. Segundo, las competencias de ciencias de la información actualmente son desarrolladas en múltiples especialidades, y este caso presenta una especialidad de negocios. Finalmente, este trabajo muestra una experiencia que podría variar en otras instancias. En el futuro, es necesario seguir explorando la utilización de nuevas herramientas y metodologías para el desarrollo de competencias de ciencias de la información. Algunos nuevos trabajos podrían abordar las diferencias entre estudiantes, trabajando con otras carreras, comparando sus resultados y generando nuevas propuestas. También evaluar las competencias mediante escalas especializadas o modelos de aceptación en muestras más grandes.

\section{CONCLUSIONES}

Este trabajo presenta la experiencia académica con el uso de Python y Django para el desarrollo rápido de prototipos de sistemas de información para estudiantes con una leve formación en el área de tecnología para las ciencias de la información. Según esto, se presentan las siguientes conclusiones: Python es un lenguaje de programación adecuado para un fácil y rápido dominio; Python es un lenguaje de programación actual con marcos de trabajo adecuados para el desarrollo rápido de soluciones informáticas; Django es una herramienta que permite la abstracción para trabajar con sistemas de base de datos (por ejemplo, no se requiere conocimiento directo de SQL); y gracias al gran desarrollo de Python como lenguaje de programación para el análisis de datos, los marcos de trabajo como Django son perfectamente integrables para el desarrollo de soluciones web para el análisis de datos.

\section{REFERENCIAS}

Aguado, J., Martínez, I., y Cañete, L.., Tendencias evolutivas del contenido digital en aplicaciones móviles, doi: 10.3145/epi.2015.nov.10, El Profesional de la Información, 24(6), Noviembre (2015)

Carneiro, T., V. Medeiros, R., y otros cuatro autores, Performance Analysis of Google Colaboratory as a Tool for Accelerating Deep Learning Applications, doi: 10.1109/ACCESS.2018.2874767, journal IEEE Access, 6, 61677 - 61685 (2018)

Chang, H., Wang, C., y Hawamdeh, S., Emerging trends in data analytics and knowledge management job market: extending KSA framework. http://doi.org/ 10.1108/JKM-02-2018-0088, Journal of Knowledge Management, 11 (2018)

CIMET, Aprendizaje + Servicio, (en línea), http://cimet.ucn.cl/as/. Acceso: 02 de Febrero (2021)

Danielsson, P., Postema, T., y Munir, H., Heroku-Based Innovative Platform for Web-Based Deployment in Product Development at Axis, https://doi.org/10.1109/ACCESS.2021.3050255, journal IEEE Access, 9, 10805 - 10819 (2021)

Gaudiot, J. -L., y Kasahara, H., Computer Education in the Age of COVID-19, https://doi.org/10.1109/MC.2020.3011277, journal Computer, 53(10), 114 - 118, Octubre (2020)

Gutiérrez-Cárdenas, J. M., Experiences in using a multiparadigm and multiprogramming approach to teach an information systems course on introduction to programming. Journal of Information Systems Education, 31(1), 72 - 82 (2020)

Haristiani, N., y Rifa'i, M.M., Combining Chatbot and Social Media: Enhancing Personal Learning Environment (PLE) in Language Learning. https://doi.org/10.17509/ijost.v5i3.28687, Indonesian Journal of Science and Technology, 5(3), 487 $-506(2020)$

IICG-UCN, Ingeniería en Información y Control de Gestión, Facultad de Economía y Administración, Universidad Católica del Norte, (en línea), http://www.eciem.cl/. Acceso: 27 de Enero (2021)

Kapoor, A., Comparative Study on Web Development using core PHP \& Laravel Framework, International Journal of Advanced Science and Technology, 29(10s), 2660 - 2663 (2020)

Kaw, J. A., Loan, N. A., y otros cuatro autores, A reversible and secure patient information hiding system for loT driven e-health, https://doi.org/10.1016/j.ijinfomgt.2018.09.008, International Journal of Information Management, 45, 262 - 275 (2019)

Koulouri, T., Stanislao, L., y Robert, M., Teaching Introductory Programming: A Quantitative Evaluation of Different Approaches, https://doi.org/10.1145/2662412, ACM Transactions on Computing Education, Association for Computing Machinery (ACM), 14(4), USA (2015)

Malla-IICG, Malla Curricular de Ingeniería en Información y Control de Gestión, Facultad de Economía y Administración, Universidad Católica del Norte, https://www.ucn.cl/. Acceso: 27 de Enero (2021) 
Martines, C., A Levels-based Approach for Defining Software Measurement Architectures, https://doi.org/ 10.19153/cleiej.17.3.4, CLElej, 17(3), Montevideo, Uruguay, Diciembre (2014)

Melé, A., Django 3 By Example: Build powerful and reliable Python web applications from scratch, Packt Publishing, 3era Ed., USA (2020)

Oliva, R., Carvajal, K., y Cataldo, A., Impacto TI en las pequeñas y medianas empresas ¿es su efecto moderado por la intensidad de uso de ti de la industria?, doi: 10.4067/S0718-27242018000200082, Journal of Technology Management \& Innovation, vol. 13, 82 - 93, Julio (2018)

Poulovassilis, A., Larsson, N., y otros tres autores, Creating a Knowledge Base to research the history of UK Museums through Rapid Application Development, https://doi.org/10.1145/3343871, Journal on Computing and Cultural Heritage (JOCCH), 12(4), 1 - 27 (2019)

Prokopyev, M. S., Vlasova, E. Z., y otros tres autores, Desarrollo de un curso de programación para estudiantes de una institución de educación superior de formación docente utilizando el lenguaje de programación Python, Propósitos y Representaciones, 8(3) (2020)

Raikar, M. M., Desai, P., y Naragund, J. G., Active learning explored in open elective course: Internet of Things (loT), doi: 10.1109/T4E.2016.012, IEEE 8th International Conference on Technology for Education (T4E), IEEE, India, 15 - 18, Diciembre (2016)

Rosenbloom, A., A simple MVC Framework for Web Development Courses, doi: 10.1145/3209635.3209637, 23rd Western Canadian Conference on Computing Education, WCCCE '18, ACM, Nueva York, USA (2018)

Salas-Zarate, M., Alor-Hernández, y otros cuatro autores, Analyzing best practices on Web development frameworks: The lift approach, http://dx.doi.org/10.1016/j.scico.2014.12.004, Science of Computer Programming, 102, 1-19, Mayo (2015)

Sánchez-Carrión, M., Coronel-Romero, E., y otros cuatro autores, Sistema informático para la gestión y publicación de la producción científica de la Universidad Nacional de Loja, Iberian Conference on Information Systems and Technologies, CISTI, 1 - 6, Junio (2018)

Schmal, R., Evolución de un programa de formación en competencias genéricas, doi: 10.4067/S071850062015000600012, Formación Universitaria, 8(6), 1 - 14 (2015)

Schmal, R., Rivero, S., y Vidal, C., El desafío de construir un programa para el desarrollo de competencias genéricas: un estudio de caso, doi: 10.1590/s1678-4634202046217017, Educação e Pesquisa, 46(7), 1 - 14 (2020)

Seo, J. Y., Lee, D. W., y Lee, H. M., Performance comparison of CRUD operations in loT based Big Data computing, https://doi.org/10.18517/ijaseit.7.5.2674, International Journal on Advanced Science, Engineering and Information Technology, 7(5), 1765-1770 (2017)

Shukla, X., y Parmar D. J., Python - A comprehensive yet free programming language for statisticians, https://doi.org/10.1080/09720510.2015.1103446, Journal of Statistics and Management Systems, Taylor \& Francis, 19(2), 277 - 284 (2016)

Tabet, N., Gedawy, H., Alshikhabobakr, H., y Razak, S., From Alice to Python. Introducing Text-based Programming in Middle Schools, doi: 10.1145/2899415.2899462, ACM Conference on Innovation and Technology in Computer Science Education, ITiCSE '16, ACM, Nueva York, USA New York, NY, USA, 124 - 129 (2016)

Tapia-Jara, J., Sánchez-Ortíz, A., y Vidal-Silva, C., Estilos de aprendizaje e intención de uso de videos académicos de YouTube en el contexto universitario chileno, doi: 10.4067/S0718-50062020000100003, Formación Universitaria, 13(1), $3-12(2020)$

Vidal, C., González, M. P., y otros tres autores, Primer curso de programación usando Python: Resultados y experiencias en Ingeniería Informática Empresarial Universidad de Talca, XI Congreso Chileno de Educación Superior en Computación (CCESC), Antofagasta, Chile, Noviembre (2010)

Zheng, J., Qin, L., y otros cuatro autores, Django: Bilateral coflow scheduling with predictive concurrent connections, https://doi.org/10.1016/j.jpdc.2021.01.006, Journal of Parallel and Distributed Computing, Febrero (2021) 